Reprod. Nutr. Dévelop., 1988, 28 (1), 105-106.

\title{
Utilisation comparée de I'ytterbium et du chrome mordancé pour mesurer le temps de séjour d'une ration chez le lapin
}

\author{
T. GIDENNE
}

Laboratoire de Recherches sur l'Elevage du Lapin,

I.N.R.A., B.P. 27, 31326 Castanet Tolosan Cedex, France.

Summary. Ytterbium was compared to chromium mordanted fiber in studying passage particule rate in rabbit. The retention time using chromium were always shorter than with Ytterbium. The origins of the differences were related to the digestive physiology of the rabbit.

Les terres rares et le chrome mordancé sont actuellement fréquemment utilisés en tant que marqueurs pour étudier le transit des particules alimentaires, mais leurs caractéristiques sont différentes. La solidité de fixation d'une terre rare peut varier en fonction de son support alimentaire, et elle diminue lorsque l'acidité du milieu digestif augmente. A l'inverse, les particules mordancées au chrome sont indigestibles et forment un complexe indissociable (Pond et al., 1986). Chez le monogastrique herbivore, ces deux marqueurs ont rarement été utilisés simultanément pour permettre leur comparaison. Nous avons donc appliqué ces deux techniques de marquage à un même aliment pour étudier son transit digestif chez le lapin.

Matériel et méthodes. De l'ytterbium $(\mathrm{Yb})$ et du chrome $(\mathrm{Cr})$ ont été fixés séparément sur du foin de luzerne débarrassé de ses constituants les plus solubles par lavage $\left(1 \mathrm{~h}\right.$ à $90^{\circ} \mathrm{C}$ avec un détergent du commerce). Le marquage par la terre rare $(\mathrm{Yb})$ a été réalisé par trempage $(24 \mathrm{~h})$ suivi d'une extraction par l'acide citrique. Le chrome est mordancé selon la technique de Uden (1978). Les deux lots de foin marqué ont été incorporés à raison de $0,4 \%$ de la MS pour l'Yb et $0,1 \%$ pour le $\mathrm{Cr}$, avant granulation dans un aliment de composition classique $(17,5 \%$ de protéines brutes et $37,2 \%$ de parois NDF), à base de luzerne déshydratée. L'aliment a été distribué ad libitum à un lot de 6 lapines adultes (Néozélandais Blanc $x$ Californien). Le taux de récupération ( $\mathrm{Tr}$ ) des deux marqueurs a été mesuré après une semaine d'accoutumance au régime, par collecte totale des fèces durant $2 \times 4$ jours. Le temps de séjour moyen (TSM) a été calculé (Faichney, 1975), par collectes fécales fractionnées sur $56 \mathrm{~h}$, à intervalles croissants ( 2 à $8 \mathrm{~h}$ ) débutant lorsque l'aliment marqué a été remplacé (à 9 h) par un aliment de même formule, mais ne contenant pas de marqueur. Le 
chrome et l'ytterbium ont été dosés dans les fèces et l'aliment par spectrométrie d'absorption atomique.

Résultats et discussion. La récupération de l'ytterbium (Yb) a été totale (tabl. 1) et celle du chrome significativement plus faible $(96,8 \%)$. Le TSM calculé à l'aide du $\mathrm{Cr}(17,7 \mathrm{~h})$ est significativement inférieur à celui mesuré avec I'Yb $(20,7 \mathrm{~h}$; tabl. 1). Selon les individus le TSM de l'Yb a dépassé de 3 à $26 \%$ celui mesuré avec le chrome, sans relation avec la quantité d'aliment ingérée (34 à $51 \mathrm{~g} / \mathrm{kg}$ poids vif). La quantité de marqueur excrétée en $24 \mathrm{~h}$ (E), soit jusqu'à la phase de caecotrophie suivante, est très élevée; elle est significativement supérieure pour le chrome.

TABL. 1. - Taux de récupération (Tr), temps de séjour moyen (TSM) et excrétion après 24 h (E) des particules marquées au $\mathrm{Cr}$ ou à l'Yb.

\begin{tabular}{lcccc}
\hline & $\begin{array}{c}\text { Concentration } \\
\text { dans l'aliment }\end{array}$ & $\begin{array}{c}\text { Tr (\%) } \\
\mathrm{n}=6\end{array}$ & $\begin{array}{c}\text { TSM (heures) } \\
\mathrm{n}=5\end{array}$ & $\begin{array}{c}\text { E (\%) } \\
\mathrm{n}=\mathbf{5}\end{array}$ \\
\hline Ytterbium & $46 \mathrm{mg} / \mathrm{Kg} \mathrm{MS}$ & $100,0 \pm 1,9$ & $20,7 \pm 1,5$ & $89,6 \pm 2,1$ \\
Chrome & $69 \mathrm{mg} / \mathrm{Kg} \mathrm{MS}$ & $96,8 \pm 1,5$ & $17,7 \pm 0,8$ & $94,6 \pm 3,3$ \\
\hline F. obs. & & $* *$ & $* *$ & $*$ \\
\hline
\end{tabular}

Chez les ruminants (Gonzalez et al., 1987) et le porc (Pond et al., 1986), le chrome conduit à des valeurs de TSM supérieures à celles obtenues avec les terres rares. Dans le cas du lapin, la situation inverse est observée, en raison probablement de la physiologie digestive particulière à cet animal. En effet, en période d'excrétion de crottes dures, le côlon proximal refoule vers le caecum les particules alimentaires de petite dimension $(<0,1 \mathrm{~mm})$, et allonge donc leur temps de séjour dans le caecum. Or, la taille des particules mordancées au chrome n'est pas susceptible d'être réduite du fait de leur indigestibilité ; elles ont par conséquent un TSM minimum dans le caecum. A l'inverse, le support de I'Yb n'est pas totalement indigestible ; la taille de ces particules peut donc être réduite, et elles sont donc susceptibles d'être refoulées en proportion plus importante, vers le caecum. De plus, nous avons mis en évidence avec ce même aliment que 5 à $10 \%$ de l'Yb fixé pouvait quitter son site initial de fixation (forte acidité stomacale...) et migrer vers d'autres particules éventuellement de faible taille (Gidenne, données non publiées). Ces deux éléments expliquent donc le TSM plus élevé mesuré avec l'Yb.

L'ytterbium et le chrome ont des comportements différents dans le tube digestif du lapin. Le choix du marqueur est donc fonction de l'information désirée : transit de particules potentiellement dégradables (Yb), ou indigestibles (Cr).

Faichney G. J., 1975. In I. W. Mc Donald et A. C. I. Warner. Digestion and metabolism in the ruminant. Proc. IV. Int. Symp. on ruminant physiology. Sidney, Australia, August 1974. $277-291$. The University of New England. Publishing Unit.

Gonzalez J., Poncet C., Michalet-Doreau B., 1987. Reprod. Nutr. Dévelop., 27, 221-222.

Pond W. G., Pond K. R., Ellis W. C., Matis J. H., 1986. J. anim. Sci., 63, 1140-1149.

Uden P., 1978. Thesis, PhD. Cornell University, 165 p. 